Ursula Bredel (Hildesheim)

\title{
Silben und Füße im Deutschen und Türkischen - Orthographieerwerb des Deutschen durch türkischsprachige Lerner/innen
}

\section{Einleitung}

Eine wichtige und häufig unterschätzte Aufgabe beim Erwerb einer zweiten Sprache ist es, sich die rhythmisch-prosodischen Muster der Zielsprache anzueignen (Kaltenbacher 1998; Gut/Trouvin/Barry 2007; Rautenberg 2012). Wie erfolgreich Lerner/innen dabei sind, ist auch davon abhängig, ob sie analoge Muster in ihrer Ausgangssprache vorfinden und damit bereits für eine zielsprachennahe Rhythmus- und Akzentwahrnehmung sensibilisiert sind. Liegen solche analogen Muster nicht vor, ist es Aufgabe von Lehrprogrammen, die Rhythmus- und Akzentstrukturen als relevante Lernhilfen sichtbar zu machen.

Soweit ich sehe, liegen hier bislang kaum zielführende Konzepte vor (so auch Richter 2008). Findet der Zweitspracherwerb in der Grundschule statt, ist sogar das Gegenteil der Fall. Denn im Schriftspracherwerb, der den weitaus intensivsten Teil des Deutschunterrichts der Grundschule ausmacht, werden die Kinder mit noch genauer zu beschreibenden Schreibund Lese-Lern-Verfahren auf die phonologische Segmentstruktur festgelegt; Rhythmus und Akzent werden nivelliert bzw., wie zu zeigen sein wird, für andere Arbeitsaufgaben funktionalisiert, so dass eine zielsprachennahe Orientierung an rhythmisch-prosodischen Strukturen tendenziell blockiert wird.

Das hat Auswirkungen nicht nur auf den Erwerb der gesprochenen Sprache, sondern auch auf den Schriftspracherwerb selbst. Denn die Schrift buchstabiert nicht einfach Lautketten aus, sondern ist selbst rhythmussensitiv. Verschriftet werden neben segmentalen Lauteigenschaften, Silbenund Akzentmuster, morphologische und syntaktische Strukturen. Eine einseitige Fixierung der Kinder auf die lautlich-segmentale Seite der Schrift führt nicht nur zu einer vereinseitigten Schrifttheorie, sondern zugleich dazu, dass weitere strukturelle Eigenschaften, darunter Silben- und Akzentmuster, weder in den Aufmerksamkeitsfokus der Kinder gelangen noch für das Schreiben und Lesen genutzt werden können. Wie u.a. Ashby (2006) gezeigt hat, ist aber gerade die Realisierung der prosodisch-rhythmischen Struktur eine wesentliche Komponente für die Leseflüssigkeit. 
Im vorliegenden Beitrag geht es nach einem Abriss über herkömmliche, im Erstunterricht zugrundegelegte Schriftspracherwerbsmodelle und ihre Folgen für die Sprachwahrnehmung um die Rekonstruktion der Wortschreibung des Deutschen; gezeigt wird, wie phonographische, silbische, prosodische und morphologische Eigenschaften im Kernwortschatz miteinander interagieren, um eine reguläre Wortschreibung zu erzeugen.

Eine Gegenüberstellung des Deutschen und des Türkischen wird zeigen, dass sowohl in Bezug auf die Akzent- als auch in Bezug auf die Rhythmusstrukturen relevante Unterschiede bestehen, die bei Kindern mit Türkisch als erster Sprache zu Problemen beim Zugriff auf die für die Verschriftung deutscher Wörter wichtigen prosodischen Strukturen führen können, die durch fehlgehende Lehr-Lernprogramme weiter verschärft werden.

Zuletzt werden neuere Modelle des Schriftspracherwerbs vorgestellt, die es auf der Grundlage einer ausgebauten Schrifttheorie erlauben, den Lerner/innen von Beginn an einen Zugriff auf diejenigen Einheiten zu ermöglichen, die Fixpunkte für das geschriebene und für das gesprochene Deutsch darstellen.

\section{Schriftspracherwerbsmodelle im Anfangsunterricht}

Schulische Lehrwerke kommen in der Regel ohne eine explizite Schrifttheorie aus. Ihre Vorgehensweisen beruhen auf der common sense-Annahme, das Deutsche sei zugrundeliegend eine Lautschrift, die geschriebene Sprache bilde - wenigstens im Prinzip - die Lautsprache ab, mithin sei die gesprochene Sprache das Modell für die geschriebene. Damit ist zugleich die Erwerbsrichtung festgelegt: Als Ausgangspunkt für den Erwerb gelten die (bereits bekannten) Laute, von denen aus die (noch unbekannten) Buchstaben erschlossen werden sollen, wobei nicht zwischen Phonen/Phonemen oder Graphen/Graphemen unterschieden wird, weshalb für die folgende Darstellung - die begriffliche Ungenauigkeit in Kauf nehmend - weiter von „Lauten“ und von „Buchstaben“ gesprochen wird.

Was die Bearbeitung von Lauten einerseits und Buchstaben andererseits angeht, werden weder die einen noch die anderen als Repräsentanten eines je eigenen medialen Systems erfasst. Vielmehr werden Buchstaben mit ihrem Lautwert so vollständig identifiziert, dass sie nicht mit ihrem Buchstabennamen, sondern mit einem artikulatorischen Lautwert eingeführt und weiterbearbeitet werden. Der Buchstabe $<\mathrm{B}, \mathrm{b}>$ heißt in der Schule nicht $B$ e, sondern [bə], $<\mathrm{F}, \mathrm{f}>$ heißt nicht Eff, sondern [f] usf.

Um zur korrekten Schreibung zu gelangen, sollen die Kinder, nachdem sie die einzelnen Laut-Buchstaben-Beziehungen in dieser Weise kennengelernt haben, in einem zweiten Schritt jedes Wort in einer Art Pilotsprache artikulieren, um ,jeden Laut herauszuhören“, und diesen dann entsprechend 
verschriften. Diese Pilotsprache ist nun nichts anderes als eine Buchstabenanalyse, was dadurch verbrämt wird, dass die Buchstaben nicht beim Namen genannt, sondern wie Laute behandelt werden. Mit der Pilotsprache wird zur Voraussetzung gemacht, was zu erwerben ist.

Die Fixierung auf die Laut-Buchstaben-Beziehung und damit einhergehend die Priorisierung des Schreibens vor dem Lesen im Anfangsunterricht macht sich auch in den theoretischen Modellierungen des Lernprozesses geltend. Das gilt sowohl für die Modellierung der so genannten Vorläuferfähigkeiten als auch für die Modellierung des Schriftspracherwerbsprozesses in den ersten Schuljahren:

Als wichtigste Vorläuferfähigkeit für den Schriftspracherwerb gilt seit ca. 20 Jahren die so genannte Phonologische Bewusstheit im engeren Sinne. Die Fähigkeit, so die Annahme, Laute aus dem Lautstrom auszugliedern, bilde einen wesentlichen Prädiktor für den Erfolg beim Schriftspracherwerb (Marx 1992). Dabei wird übersehen, dass die segmentale Lautanalyse in Wahrheit Schriftanalyse ist. Sie bildet sich nicht vor dem Eintritt in den Schriftspracherwerbsprozess heraus, sondern ist die Folge der Auseinandersetzung mit der Schrift (Huneke 2008; Birk/Häffner 2005). Ein Training der Phonologischen Bewusstheit zur Vorbereitung auf den Schriftspracherwerb, wie es beispielsweise von Schneider/Roth/Küspert (1999) vorgeschlagen und vielfach praktiziert wird, ist somit vorgezogener Schriftspracherwerb, nicht seine Vorbereitung.

Die Annahme, die Ausgliederung von Lauten stelle den Ausgangspunkt im Schriftspracherwerb dar, ist auch modellbildend für die Konzeptualisierung von Entwicklungspfaden des Schriftspracherwerbsprozesses, die seit den 1980er Jahren nach dem Vorbild Piagets den Charakter von Phasenmodellen haben. Das bekannteste ist wohl das Modell der Psychologin Uta Frith (1985), die davon ausgeht, eine zunächst zu beobachtende logographematische Phase (ganzheitliche Symbolerkennung) werde von einer alphabetischen Phase (Orientierung am Einzellaut) abgelöst, auf die dann eine orthographische Phase (Orientierung an Schreibnormen) folge. In den Folgejahren wurden mit zum Teil erheblichen Differenzierungen weitere solcher Phasenmodelle zur Diskussion gestellt (z.B. Valtin 1997; Thomé 1999, 2003; Scheerer-Neumann 1998; zu einem Überblick Becker 2008). Über alle Unterschiede im Detail hinweg weisen diese Modelle eine Gemeinsamkeit auf: Sie konzipieren den Schriftspracherwerbsprozess unter Absehung des Unterrichtsangebots, das zu den entsprechenden Schreib- und Leseresultaten führt, die als Indikatoren für die Zuordnung zu einer Phase gelten. So läuft die gesamte Schriftspracherwerbsforschung Gefahr, als Ontologie zu modellieren, was in Wahrheit instruktionsbedingt ist. Nicht zufällig sehen alle Modelle die Orientierung am Einzellaut als Eintrittskarte in die Schrift, also genau derjenigen Einheit, mit der die schulische Instruktion startet. 
Die quasireifungstheoretischen Modelle bilden nun umgekehrt auch die Grundlage für die Absicherung der Didaktisierung des Anfangsunterrichts, der seine zentrale Aufgabe darin sieht, die „erste Phase“, also die Lautwahrnehmung anzustoßen. Es entsteht ein nicht aufzulösender circulus vitiosus, der auch Konsequenzen für die Analyse misslungener Schriftspracherwerbsbiographien hat: Kindern, die einen verlangsamten Eintritt in die nächste „Phase“ zeigen, wird nicht selten eine verzögerte Individualentwicklung bescheinigt; das Unterrichtsangebot als auslösender Faktor kommt auf der Basis eines als „natürlich“ gedachten Erwerbsprozesses nicht in Betracht.

Flankiert wird der hier dargestellte Zirkel durch allgemeine lerntheoretische Grundannahmen, die ebenfalls seit den 1980er Jahren das Bild des Lernens in der Grundschule prägen: In Abgrenzung zu behavioristischen Lernmodellen, in denen das Lernangebot von außen als Input und zugleich als Intake für Lernprozesse gesehen wird, führte die Adaption konstruktivistischer Theoreme dazu, das Lernen als eigenaktiven Prozess zu modellieren. In Bezug auf den Schriftspracherwerbsprozess spricht Eichler bereits 1976 von der ,inneren Regelbildung“. Gemeint ist, dass Kinder im Lernprozess den Input eigenständig aufgreifen und im selbstständigen Umgang mit der Schrift deren Gesetzmäßigkeiten entdecken. In der Didaktik hat dieser zunächst produktive Begriff im Konzert mit der bereits benannten quasireifungstheoretischen Auffassung eine unglückliche Auslegung erfahren: Man meinte daraus ableiten zu können, dass Schreiben- und Lesenlernen letztlich nicht gesteuert, sondern als ,individuelle“ Vorgänge lediglich begleitet werden könnten. Gestützt wird diese Auffassung wiederum durch allgemeinpädagogische Trends, die - übrigens ganz im Widerspruch zu den als überindividuell konzipierten Phasenmodellen - dem Individuum und dem individuellen Lernen den Vorrang vor dem gemeinsamen Lernen geben. Eine Auffassung, die ihre Wurzeln in der Reformpädagogik des beginnenden 20. Jahrhunderts hat und die mit den beginnenden 1980er Jahren bildungspolitisch reanimiert wurde. Die Folgen für den Anfangsunterricht sind dann häufig so genannte freie Lernangebote, offener Unterricht und die Förderung der so genannten Kreativität. Auf eine (Vor-)Strukturierung des Lernangebots wird weitgehend verzichtet.

Ein Modell, in dem geradezu idealtypisch alle Komponenten des lerntheoretischen Diskurses der vergangenen 30 Jahre gebündelt sind, und das deshalb als „,vorläufige[r] Höhepunkt in der didaktischen Bewegung“ (Blumenstock 1993, S. 90) gesehen wurde, stellt das Programm „Lesen durch Schreiben" von Jürgen Reichen dar, das sich nicht zufällig mit den beginnenden 1980er Jahren epidemisch an Schulen verbreitete und das an Schlichtheit unübertroffen ist: Die Kinder erhalten zu Beginn des Schriftspracherwerbsprozesses eine so genannte Anlauttabelle mit Bild-Buchstabenpaaren. Der Anfangsbuchstabe des Wortes, das den jeweils abgebildeten Gegenstand bezeichnet, ist mit dem dem Bild zugeordneten Buchstaben identisch. Durch 
genaues Hinhören und genaues Aussprechen sollen Laut für Laut eines Wortes aufgespürt und der entsprechende Buchstabe dafür notiert werden. Ganz im Sinne der Phasentheorie wird nicht erwartet, dass ein Wort „orthographisch korrekt geschrieben wird; es genügt zunächst, wenn es lautgetreu, d.h. phonetisch vollständig notiert [...] wird“ (Reichen 2001, S. 27). Und dem reifungstheoretisch fundierten Konstruktivismus folgend geht Reichen davon aus, dass „Lernprozesse didaktisch gesehen nur begrenzt gesteuert und kontrolliert werden" können (ebd., S. 20) und definiert Lernen als etwas, „was jedes Kind alleine schafft (oder eben nicht)“ (ebd.). Dem Primat des Schreibens vor dem Lesen folgend wird auf das Lesen nicht nur vollständig verzichtet, es wird vielmehr für schädlich gehalten; so meint Reichen, es sei „wichtig, dass im Verlauf des Lernprozesses kein Kind jemals gezwungen wird, etwas zu lesen“ (Reichen 1988, S. 8). Weiter heißt es: „Wer gegen Ende des III. Quartals noch nicht liest, soll mehr schreiben“ (ebd., S. 48).

Vergleichsstudien, die seit den beginnenden 1990er Jahren die Wirksamkeit von „Lesen durch Schreiben“ im Vergleich mit lehrgangsgebundenen Modellen untersuchen, erhalten alle denselben Befund: Die mit „Lesen durch Schreiben" beschulten Kinder waren überall die schwächsten (vgl. Metze 2008). Den Schulalltag scheinen solche Befunde nicht zu belasten: Wie Störmer (2008) gezeigt hat, fehlt die Anlauttabelle in fast keiner Grundschulklasse.

An einem eher mikroskopischen Beispiel soll hier gezeigt werden, welche Schwierigkeiten bei der Verschriftung auf der Grundlage der durch die Anlauttabelle gegebenen Informationen über die Schrift entstehen. Abbildungen 1 und 2 zeigen ein Schreib- und ein Lesenotat von Robert, der versucht, sich das Wort Gurke zu erschließen (Schreibnotat, Abb. 1 in Anlehnung an Vogt 2004, S. 131). Die Notate zeigen auch, warum viele Kinder nicht lesen können, was sie geschrieben haben.
$\begin{array}{llll}0 & 1 & 2 & 3\end{array}$
$\begin{array}{lll}4 & 5 & 6\end{array}$
$\begin{array}{ll}7 & 8\end{array}$
9

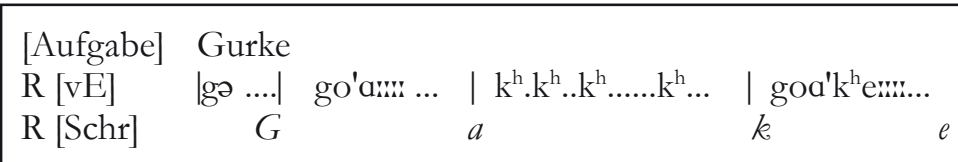

Abb. 1: Schreibnotat eines Erstklässers

$$
\begin{aligned}
& \text { [Aufgabe] }<\text { Gake }>\text { (Eigenschreibung für }<\text { Gurke }>\text { ) }
\end{aligned}
$$

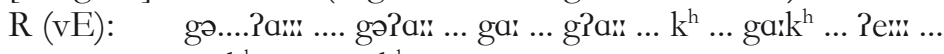

$$
\begin{aligned}
& \text { ga:khew: ... ga:khe: }
\end{aligned}
$$

Abb. 2: Lesenotat eines Erstklässers 
Beim Schreiben können wir folgende Verfahren beobachten: Die Konsonanten werden von Robert koartikuliert, das stimmhafte [g] wird zu einer Schwasilbe ausgebaut, das stimmhafte $[\mathrm{k}]$ aspiriert. Die Verschriftung der reinen Konsonantbuchstaben $<\mathrm{g}>$ und $<\mathrm{k}>$ zeigt jedoch, dass Robert hier bereits eine Abstraktionsleistung vollzogen hat und zwischen Kern- und Koartikulation unterscheidet. Erheblich größere Probleme bereiten Robert die Vokale: Sie werden, gemäß der Pilotsprache, in ihrer gespannten Langversion artikuliert. Beim öffnenden Diphthong [ue] wird die Artikulation während dieser Überdehnung auf den Zweitbestandteil verlagert; die ,genaue Aussprache" führt so zur Herausarbeitung von [a:], das mit $<a>$ verschriftet wird. Einen sehr deutlichen Hinweis darauf, wie die Pilotsprache die Normalaussprache überlagert, gibt die Bearbeitung des zweiten Silbennukleus: Robert gibt dem Reduktionsvokal die Vollartikulation [e:]; er überschreibt seine Normalaussprache, um zum Buchstaben $\langle\mathrm{e}\rangle$ zu gelangen.

Die Akzentgebung ist in die Erschließung der vokalischen Nuklei eingespannt. Robert akzentuiert jeweils dort, wo er eine Laut-BuchstabenZuordnung vornimmt. Ich spreche von einem Arbeitsakzent.

Auch beim Lesen wird der Arbeitsakzent aktiv: Robert akzentuiert bei der Lautsynthese beide vokalischen Kerne. Sein Leseresultat, die Folge von zwei akzentuierten Silben, entspricht so nicht der trochäischen Normalaussprache. Dass Robert nicht versucht, die Wortform [ga:ke:] mit einem Lexikoneintrag zu verbinden, gibt auch einen Einblick in seine Lesetheorie: Für ihn ist Lesen ganz offenbar die vollständige Zusammenfügung (,Synthese") von in Buchstaben abgelegten Lautwerten, nicht die Erfassung einer Bedeutung. Kinder, die den Sprung in die Strukturanalyse statt des linearen Abarbeitens von Buchstabenketten nicht meistern, erkennen wir häufig noch weit über den Anfangsunterricht hinaus daran, dass sie sich durch ganze Sätze und Texte regelrecht hindurchfuhrwerken, ohne dass sie versuchen, den Sinn zu erfassen (vgl. Noack 2004).

\section{Die Wortschreibung des Deutschen}

Die genauere Analyse der Schreib- und Leseprobe von Robert hat gezeigt, dass erhebliche Unterschiede zwischen dem Konsonantismus und dem Vokalismus bestehen: Während Robert bei der Konsonantenverschriftung und -lesung zu zielsprachennahen Repräsentationen gelangt ist, stellen sich bei den Vokalen in beiden Modi erhebliche Probleme ein. Das betrifft zum einen, wie bereits angesprochen, den Wortakzent, der zugunsten des Arbeitsakzents neutralisiert wird, zum anderen betrifft es die Vokalqualität/ -quantität.

Wie schon häufig festegestellt wurde, ist das System der Vokalbuchstaben im Deutschen unterspezifiziert. Akzeptiert man die reguläre $<$ ie $>$-Schrei- 
bung als Basisgraphem für [ii] (Thomé 1999) und lässt die Diphthonge zunächst unberücksichtigt (vgl. dazu umfassend Berg/Fuhrhop 2011), stehen 9 Vokalgrapheme mindestens 16 Vokalen gegenüber.

Allein dieser Befund zeigt, dass die Wortschreibung des Deutschen nicht allein über die Phonographie erklärt ist. Für eine systematische Rekonstruktion des Systems der Wortschreibung, die es erlaubt, die Vokalqualität/-quantität vorauszusagen, ist vielmehr eine hierarchische, nicht-lineare Struktur anzunehmen, wie sie in der Linguistik seit längerem diskutiert wird (vgl. z.B. Maas 1992; Weingarten 2004).

Den Ausgangspunkt eines hierarchischen Modells des graphematischen Wortes bildet der trochäische Fuß (kaufen, Gurke, flattern, Spinne), das prosodische Basismuster des deutschen Kernwortschatzes. Abbildung 3 stellt eine mögliche Modellierung dar:

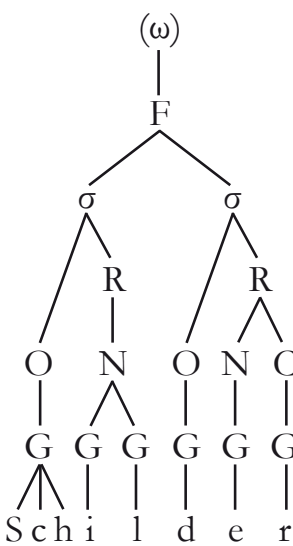

$$
\begin{aligned}
& \text { graphematisches Wort } \\
& \text { graphematischer Fuß } \\
& \text { graphematische Silben } \\
& \text { graphematische Silbenkonstituenten: } \\
& \mathrm{R}=\text { Reim, } \mathrm{O}=\text { Onset, } \mathrm{N}=\text { Nukleus, } \\
& \mathrm{C}=\text { Coda } \\
& \text { Grapheme } \\
& \text { Buchstaben }
\end{aligned}
$$

Abb. 3: Konstituentenstruktur des graphematischen Wortes (nach Primus/Evertz 2011)

Die Auswertung der (terminalen) Buchstabenfolge führt dieser Darstellung zufolge nicht direkt zu einer phonologischen Repräsentation und damit zu einem lexikalischen Eintrag, sondern über die Ermittlung des Graphemstatus zur Bildung von Silben und Füßen mit kanonischer Akzentstruktur und von dort aus zur Zuordnung eines Lautwertes. Erst so wird vorhersagbar, welcher vokalische Wert von einem Vokalbuchstaben kodiert ist:

Die zweite, stets unbetonte Silbe (Reduktionssilbe) weist regelhaft den Buchstaben $\langle\mathrm{e}\rangle$ im Nukleus auf. Es handelt sich jedoch keinesfalls um eine phonographische Abbildung von [e:] oder $[\varepsilon]$, sondern um einen optischen Marker, der das Vorliegen einer (unbetonten) Silbe indiziert. Seine phonologische Repräsentation kann dem nuklearen $<\mathrm{e}>$ der Reduktionssilbe erst in Relation mit dem Buchstaben der Reduktionssilbenkoda zugewiesen werden. Im vorliegenden Fall resultiert aus der Verrechnung des Nukleus $<\mathrm{e}>$ mit der Koda $<\mathrm{r}>$ das tiefe Schwa [e]. 
Bei der ersten, betonten Silbe, die im Gegensatz zur Reduktionssilbe einen komplexen Nukleus aufweist, liegen die Verhältnisse wie folgt: Ein nicht-verzweigender Nukleus (La-den, ge-ben) indiziert einen gespannten Langvokal, ein verzweigender Nukleus (lan-den, gel-ben) indiziert i.d.R. einen ungespannten Kurzvokal. Soll ein Kurzvokal erzeugt werden, ohne dass hinreichendes phonologisches Material zur Verfügung steht, wird der internukleare Konsonantbuchstabe verdoppelt (Klad-de, Eb-be). Phonologisch entspricht dies einem Silbengelenk (Eisenberg 2006), graphematisch entsteht ein verzweigender Nukleus (Primus/Evertz 2011).

Zwei weitere orthographische Markierungen weisen die Silbe als zentrale Zugriffsgröße aus: Das silbeninitiale h (flehen, Mühe) und das Dehnungs-h (Koble, feblen). Das silbeninitiale h indiziert die Silbengrenze, die ansonsten wegen des adjazenten Kontakts von zwei Vokalbuchstaben unmarkiert bliebe; das Dehnungs-h stellt sicher, dass der folgende Konsonant (n, m, r, l) nicht die Coda der Vollsilbe, sondern den Onset der Reduktionssilbe belegt. Man spricht deshalb auch vom silbenschließenden h.

Mit diesen fünf orthographischen Mustern (offene Hauptsilbe, geschlossene Hauptsilbe, Doppelkonsonantschreibung, silbeninitiales h und Dehnungs-h) sind alle silben- und fußbasierten orthographischen Gesetzmäßigkeiten der Kernwortschreibung erfasst.

Die Ausbuchstabierung der morphologischen Struktur verlangt jedoch nach einer weiteren analytischen Differenzierung. Im Deutschen gilt das so genannte Stammkonstanzprinzip: Stämme werden in allen Umgebungen so ähnlich wie möglich geschrieben. Silben- und Stamm- bzw. Morphemgrenzen sind nicht deckungsgleich; wohl aber ist die Position der Morphemgrenze - ausgehend von dem Modell in Abbildung 3 - vorhersagbar: Sie liegt zwischen dem Onset und dem Reim der Reduktionssilbe. Das in Abbildung 3 gezeigte Grundmuster kann in Bezug auf die Stammkonstanzschreibung also wie in Abbildung 4 dargestellt erweitert werden.

Wir schreiben deshalb <kommt $>$ wg. $<$ komm $\mid$ en $>$, < geht $>$ wg. $<$ geh $\mid$ en $>,<$ legt $>$ wg. $<\operatorname{leg} \mid$ en $>,<$ Schild $>$ wg. $<$ Schild $\mid$ er $>$ usw. Mit phonographischen Repräsentationen haben diese Strukturen nur noch sehr vermittelt zu tun.

Wird den Lerner/innen der Einblick in die Hierarchie des graphematischen Wortes durch eine Fixierung auf Laut-Buchstaben-Beziehungen verwehrt, schneidet man ihren Lese-Schreib-Erwerb systematisch von der erforderlichen kognitiven Durchdringung des Sprachsystems ab und versperrt ihnen wichtige Einsichten in den Bau der Schrift und der Sprache.

Die Probleme mit einem eindimensionalen Schriftspracherwerbskonzept vervielfachen sich bei Kindern mit Deutsch als zweiter Sprache: Denn sie verfügen nicht notwendig über die sprachstrukturellen Muster, die zur Aufschlüsselung der Schriftsprache (auch ohne Unterstützung durch Lernprogramme) aktiviert werden müssen. 


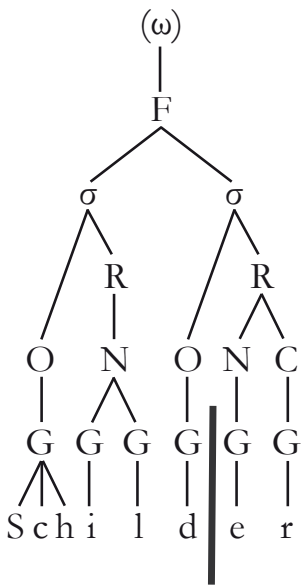

Morphemschnitt graphematisches Wort

graphematischer Fuß

graphematische Silben

graphematische Silbenkonstituenten:

$\mathrm{R}=$ Reim, $\mathrm{O}=$ Onset, $\mathrm{N}=$ Nukleus,

$\mathrm{C}=\mathrm{Coda}$

Grapheme

Buchstaben

Abb. 4: Silben- und Morphemschnitt

Bei türkischen Lerner/innen gilt dies vor allem für Silben- und Fußstrukturen. Eine Rekonstruktion der typologischen Unterschiede zwischen deutschen und türkischen Rhythmus- und Akzentmustern kann zeigen, welche Hürden türkische Lerner/innen auf dem Weg zur Schrifterkennung nehmen müssen.

\section{Rhythmus und Akzent im Deutschen und im Türkischen}

Typologisch wird unterschieden zwischen Sprachen mit gebundenem, d.h. tendenziell vorhersagebarem Wortakzent, und Sprachen mit freiem, d.h. tendenziell distinktivem Wortakzent. Das Türkische gehört zum ersten Typ; es präferiert Ultimabetonungen, weist also tendenziell eine jambische Struktur auf (zu Wörtern mit lexikalischem Akzent im Türkischen vgl. Domahs et al. 2012). Bei Sprachen mit gebundenem Akzent ist es gleichgültig, ob der Akzent auf ein Stammmorphem oder ein grammatisches Morphem fällt. Domahs et al. (2012) stellen die Regularität des türkischen Wortakzents an folgender Beispielkette dar:

ki'tap

kitap'lar

kitaplar'ım

kitaplarım'da

kitaplarımda'ki

kitaplarımdaki'ler

kitaplarımdakıler'e
Buch

Bücher

meine Bücher

in meinen Büchern

der in meinen Büchern

die in meinen Büchern

zu denen in meinen Büchern 
In Sprachen mit freiem Wortakzent (z.B. Russisch) wird die Betonung lexikalisch distinktiv genutzt. Das Deutsche nutzt den Wortakzent zur Bedeutungsunterscheidung nur in wenigen Fällen (z.B. bei der Unterscheidung von Partikel- und Präfixverben des Typs ' um-fahren vs. um-' fahren) und gilt deshalb häufig typologisch als Zwischentyp (Wängler 1974). Im Kernwortschatz ist jedoch eine deutlich morphologische Komponente erkennbar: Betont wird der Stamm, die rechts- und linksadjazenten funktionalen Morpheme sind unbetont (' fah-ren, Be-' such), wobei die linksadjazenten, unbetonten Morpheme in der Regel Derivationsmorpheme sind. Eine jambische Struktur (Be-'such) verweist im Deutschen mithin auf Wortbildung. Eine trochäische Struktur verweist auf Grundformen (' Tan-te) oder auf flektierte Formen ('Stif-te). An diesen setzt, wie oben gezeigt, die Orthographie an.

Eine zweite typologische Klassenbildung wird für die Unterscheidung zwischen dem Deutschen und dem Türkischen relevant: Das Deutsche gehört zur Familie der akzentbasierten Sprachen, das Türkische zur Familie der silbenbasierten Sprachen. In akzentbasierten Sprachen ist die Abfolge von Akzenten rhythmusstiftend. In silbenbasierten Sprachen ist die Quantität von Silben ausschlaggebend für die rhythmische Struktur (Abercrombie 1967). Die neuere Forschung hat immer deutlicher herausgearbeitet, dass die verschiedenen Rhythmuswahrnehmungen nicht auf einer einzelnen, phonetischen Eigenschaft (Isochronie von Akzenten bzw. Silben), sondern auf einem Bündel von phonologischen Merkmalen beruhen (Dauer 1983; Auer/Uhmann 1988; Auer 2001; Richter 2008):

\begin{tabular}{ll}
\hline $\begin{array}{l}\text { silbenbasierte Sprachen } \\
\text { (z.B. Türkisch) }\end{array}$ & $\begin{array}{l}\text { akzentbasierte Sprachen } \\
\text { (z.B. Deutsch) }\end{array}$ \\
\hline einfache Silbenstruktur (präferiert CV) & komplexe Silbenstruktur \\
\hline klare Silbengrenzen & Ambisilbizität \\
\hline keine Vokalreduktion & Vokalreduktion \\
\hline keine distinktive Vokalquantität & distinktive Vokalquantität \\
\hline Vokalharmonie möglich & keine Vokalharmonie \\
\hline keine schwachtonigen Funktionswörter & schwachtonige Funktionswörter \\
\hline
\end{tabular}

Tab. 1: Silbenzählende und akzentzählende Sprachen

Die Spracherwerbsforschung der vergangenen gut 20 Jahre hat Rhythmusund Akzentmuster zunehmend als Schlüssel für die Ermittlung auch lexikalischer und grammatischer Strukturen durch Lerner/innen entdeckt:

So ist seit längerem bekannt, dass die Betonungsmuster in der Phase des kanonischen und bunten Babbelns, also noch vor Eintritt in den Wortschatzerwerb bereits die zielsprachenspezifischen Betonungsmuster aufwei- 
sen: Türkische Kinder babbeln bevorzugt mit Ultimabetonung, deutsche mit Pänultimabetonung. Penner (2007) spricht im Zusammenhang mit der Herausarbeitung der zielsprachenkonformen Akzentmuster zu Recht von „Gussformen“, in die im späteren Erwerb die Wörter eingetragen werden.

Bekannt ist auch, dass Kinder bei der Ermittlung des Wortmaterials einer Sprache die Betonung als Indikator für Wortgrenzen nutzen. Im Deutschen gilt Betontheit - grosso modo - als Indikator für eine wortinitiale Position (Weber et al. 2004), in der Produktion werden Amphibrachien auf Trochäen verkürzt (Manda' rine $\rightarrow$ ' rine, $B a^{\prime}$ nane $\rightarrow$ ' nane). Türkische Kinder nutzen die wortfinale Position als Indikator für Wortgrenzen.

Auch zur Ermittlung der Wortstruktur greifen deutsche und türkische Kinder auf verschiedene Informationen aus dem Input zurück: Während für deutsche Kinder Betontheit als Indikator für lexikalisches Material und Unbetontheit als Indikator für grammatisches Material gelten, schlüsseln Kinder mit türkischer Erstsprache die interne Wortstruktur auf der Grundlage des Vokalismus auf: Vokalische Varianz (Vokalharmonie) gilt als Indikator für grammatisches Material, vokalische Stabilität als Indikator für lexikalisches Material.

Neben dem Erschließen der Strukturen von Wörtern ist zugleich die Inventarisierung und Verarbeitung bereits angeeigneten Wortmaterials von Bedeutung. In verschiedenen Studien wurde ermittelt, dass prosodischrhythmische Analogien das Speichern von lexikalischen Einheiten begünstigen (Weinert 1996). Durch Strukturähnlichkeiten im Wortmaterial werden zusätzlich die Speicherkapazität (Grimm 2003) und die Effizienz der Informationsverarbeitung erhöht (Glück/Obergföll 2009).

Ein weiterer Befund im Zusammenhang mit der Wahrnehmung und Nutzung von Akzenten ist für uns von außerordentlichem Interesse: Für Sprecher mit Französisch als Erstsprache, einer Sprache mit gebundenem Wortakzent, die Spanisch, eine Sprache mit freiem Wortakzent lernten, ermittelten Dupoux et al. (1997) eine Form von „Betonungstaubheit“ - die Lerner/innen waren nur bedingt in der Lage, den spanischen Wortakzent wahrzunehmen. Vorhersagbare Akzente scheinen auch bei der Wahrnehmung der Erstsprache nur bedingt bewusstheitsfähig zu sein. So konnten Domahs et al. (2012) zeigen, dass türkische Muttersprachler/innen abweichende Wortakzente bei nativen Wörtern mit lexikalischem Akzent sicherer detektieren als abweichende Wortakzente bei nativen Wörtern mit gebundenem Akzent. 


\section{Der Schriftspracherwerb von Kindern mit Türkisch als Erstsprache}

Spätestens in den 1970er Jahren war die Erkenntnis nicht mehr abzuweisen, dass die Kinder der so genannten „Gastarbeiter“, die Deutsch nicht als Erstsprache erworben hatten, im deutschen Schulsystem signifikant geringeren Erfolg hatten als Kinder mit deutscher Muttersprache. Das Essener Projekt zur sprachlichen Entwicklung türkischer Schüler kann als einer der wichtigen Startpunkte zur Erforschung der Sprachentwicklung türkischer Schüler/innen in Deutschland gelten, das seine Aufgabe auch darin sah, Lehrern und Lehrerinnen gesicherte Wissensbestände für eine optimierte Sprachförderung zur Verfügung zu stellen. Die in diesem Zusammenhang entstandene Publikation von Meyer-Ingewersen/Neumann/Kummer (1977) bezieht den Erwerb der Schriftsprache explizit ein. Ermittelt werden, im Sinne einer kontrastiven Erwerbshypothese, überwiegend Fehler, die durch einen oberflächennahen Transfer vom Türkischen auf das Deutsche entstehen können (z.B. Auslassung von Reduktionsvokalbuchstaben $(*<$ Bsuch $>$ für $<$ Besuch $>$ ) und/oder vokalharmonische Verschriftung $(*<$ Glızın $>$ für $<$ gelesen>, Pracht 2012, S. 19), Einfügen von Sprossvokalbuchstaben $(*<$ Birif $>$ für $<$ Brief $>)$, Nichtrealisierung der Gelenkschreibung $(*<$ falen $>$ für $<$ fallen $>)$ ).

Eine veränderte Sicht auf (Schrift-)Spracherwerbsprofile wurde in den 1980er Jahren mit der Interlanguagehypothese eingeleitet. Fehler in der Zielsprache wurden nicht mehr als Folgen eines unmittelbaren Transfers aus der Erstsprache, sondern als autonome Lernerhypothesen über die Schrift gedeutet. Damit war es auch möglich, Schreibweisen zu erklären, für die es weder in der Erst- noch in der Zweitsprache analoge Muster gibt. Auf der Grundlage der Interlanguagehypothese sortierte Thomé (1987) die Fehlschreibungen von Erst- und Zweitsprachensprecher/innen danach, welche Probleme tatsächlich nur bei Zweitsprachensprecher/innen vorkommen und deshalb tatsächlich als Interferenzen aufzufassen waren, und welche Fehler in beiden Gruppen vorkamen und darauf zurückzuführen sind, dass sowohl Erst- als auch Zweitsprachensprecher sich eine neue sprachliche Modalität aneignen müssen, für die sie z.T. eigene Hypothesen ausbilden. Im Ergebnis, so stellte Thomé fest, waren lediglich zwischen 2,7\% und 4,8\% eindeutig als Interferenzfehler identifizierbar.

Zu ähnlichen Ergebnissen kommt Becker (2011), die ermittelt hat, dass die Fehlschreibungen von Kindern mit Türkisch als erster Sprache nicht qualitativ, wohl aber quantitativ von den Fehlschreibungen von Kindern mit Deutsch als erster Sprache abweichen. Die Hauptschwierigkeiten scheinen in den Schärfungs- und Dehnungsmarkierungen zu liegen, also in der Verschriftung des Vokalismus (vgl. dazu auch Steinig et al. 2009). 
Dies hat, so die hier vertretene These, nur sehr bedingt mit einem Transfer von der Erst- auf die Zweitsprache zu tun, ist aber auch nicht unabhängig von der Erstsprache: In Anlehnung an Dupoux et al. (1997) gehe ich davon aus, dass Kinder mit Türkisch als Erstsprache wegen der schwächer ausgeprägten Akzentwahrnehmung (Betonungstaubheit) - einen erschwerten Zugriff auf silben- und akzentbedingte Schreibungen und damit auf die Verschriftung des Vokalismus (Dehnung/Schärfung) haben.

Dass auch Kinder mit Deutsch als Erstsprache bei der Wortschreibung am intensivsten mit den Dehnungs- und Schärfungsmarkierungen zu kämpfen haben, verweist nun aber auf ein tiefergehendes Problem, das in Kapitel 2 bereits angesprochen worden ist: Der herkömmliche Unterricht gestattet es weder Kindern mit Deutsch noch Kindern mit Türkisch als Erstsprache, die rhythmisch-prosodischen Muster des Deutschen als schreibund leserelevante Größen wahrzunehmen und zu nutzen. Mit der Fixierung auf die segmentale Lautseite der Schrift und durch die Aktivierung der Pilotsprache wird der Akzent der Normalaussprache nicht nur unterdrückt, sondern durch den Arbeitsakzent (siehe oben) überschrieben.

Dass Kinder mit Deutsch als Erstsprache dennoch tendenziell besser abschneiden als Kinder mit Türkisch als Erstsprache, ist möglicherweise dadurch bedingt, dass sie unter Absehung der Unterrichtsinstruktionen ihr rhythmisch-prosodisches Wissen aktivieren können, über das Kinder mit Türkisch als Erstsprache nachweislich nicht in demselben Maße verfügen.

Ganz in diesem Sinne resümiert Becker (2011) ihre Ergebnisse: Lerner/ innen mit Türkisch als Erstsprache, so die Autorin, greifen beim Schriftspracherwerb eher auf resultative Ressourcen zurück, nutzen also - im Sinne der Analogiebildung - stärker bereits angeeignetes Wissen z.B. über Wortbilder; Lerner/innen mit Deutsch als Erstsprache orientieren sich stärker an sprachlichen Regularitäten, die ihnen aus ihrer Muttersprache vertraut sind.

\section{Rhythmus und Akzent sehen lernen}

Wie in den vorangegangenen Kapiteln deutlich geworden ist, kann eine Didaktik, die Buchstaben einseitig als Abbilder von Lauten interpretiert, weder Kindern mit Deutsch als Erstsprache noch Kindern mit einer anderen Erstsprache gerecht werden. Bei Kindern mit Türkisch als erster Sprache macht sich dieser didaktische Kunstfehler jedoch stärker bemerkbar als bei Kindern mit Deutsch als Erstsprache, weil sie über die Betonungsmuster zur Aufschlüsselung der orthographischen Verhältnisse im Deutschen nicht hinreichend verfügen.

Nun sind in den vergangenen gut 15 Jahren Schriftspracherwerbsmodelle entwickelt worden, die es den Lerner/innen erlauben, von Beginn an die rhythmisch-prosodischen Strukturen für die Verschriftung zu nutzen. 
Im Fokus standen zunächst nicht Kinder mit nichtdeutschen Herkunftssprachen. Inspiriert waren diese Neuentwicklungen vielmehr durch Entwicklungen der Sprachwissenschaft, die die Schrift seit den 1980er Jahren immer deutlicher als autonomes sprachliches Teilsystem konturiert hat. Beim Eintritt in die Schrift, so die moderne Sprachdidaktik, muss nicht eine Übersetzung der gesprochenen in die geschriebene Sprache geleistet werden; vielmehr muss die Schrift als autonomes Teilsystem mit eigenen Gesetzmäßigkeiten erschlossen werden.

Trotz großer Unterschiede im Detail wählen alle alternativen Lernprogramme das trochäische Basismuster als Ausgangspunkt. Es bildet nicht nur die Grundlage für die Orthographie (vgl. Kap. 3), sondern ist zugleich dasjenige Muster, auf das Kinder mit Deutsch als Erstsprache in der Regel besonders gut zugreifen können. Wie diese Modelle gerade den Erwerb der deutschen Orthographie auch bei Kindern mit Türkisch als Erstsprache unterstützen können, soll im Folgenden skizziert werden. Dabei sehe ich von schrifttheoretischen Differenzen der Einzelvorschläge weitgehend ab. Dargestellt werden sollen die grundsätzlichen Zugriffsweisen der Modelle auf die Schreibung des Deutschen.

Hinney $(1997,2010)$ modelliert den Rechtschreibunterricht auf der Grundlage von Schlüsselwörtern (Trochäen), in denen die phonologischen Informationen der Schreibsilbe systematisch aufgefunden werden können; die morphologischen Vererbungen (Stammkonstanz) werden darauf aufbauend operativ erschlossen.

Graphisch wird die trochäische Struktur durch Silbenbögen anschaulich gemacht, die morphologische Ableitung wird mit einer eigenen optischen Form davon abgegrenzt (Abb. 5). Die klare Unterscheidung zwischen basalen, trochäischen Wortschreibungen und den darauf aufbauenden morphologischen Mustern wird im Unterricht als ein Nacheinander modelliert, wobei silbische und morphologische Informationen mit zunehmender Leseund Schreiberfahrung auch integrativ genutzt werden.

Eine wichtige Rolle spielt bei Hinney der Aufbau metasprachlichen Wissens, das es den Schüler/innen gestattet, die eigenen Wortschreibungen kriteriengeleitet zu überprüfen. Im herkömmlichen, lautbasierten Unterricht hilft hier nur das Wörterbuch.

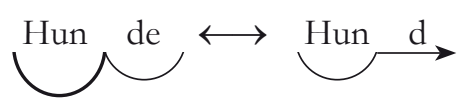

Abb. 5: Visualisierung von Silben-/Fuß- und Wortstrukturen nach Hinney (1997)

Für Kinder mit Deutsch als Zweitsprache sind mindestens drei Komponenten des Modells von Hinney (1997) besonders lernförderlich: Die Visualisierung des trochäischen Grundmusters gestattet es den Kindern, die typischen Wortstrukturen des Deutschen zu entdecken und kognitiv zu 
verstetigen. Die analytische Herangehensweise erlaubt eine systematische Auswertung von Silbeneigenschaften. Das Moment der Sprachbewusstheit, das gerade im Zweitspracherwerb eine große Rolle spielt (Jeuk 2007), ist konstitutiver Teil des Programms.

Auch bei Röber-Siekmeyer (1997) und Röber (2009) steht der trochäische Fuß am Anfang. Er wird für Lerner/innen im Bild eines Hauses (für die betonte Silbe) und einer Garage (für die unbetonte Silbe) visualisiert, in denen jeweils der Onset und der Reim von Haupt- und Reduktionssilbe abgebildet sind:

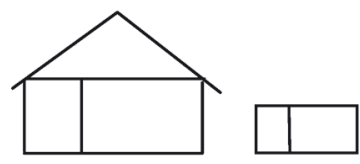

Abb. 6: Didaktisches Basismuster nach Röber (2009)

Für Kinder mit Türkisch als Ausgangssprache ist diese Visualisierung von außerordentlichem Nutzen: Denn sie können den typischen Wortakzent des Deutschen, das trochäische Muster, sehen lernen. Zusätzlich gestattet das Modell auch die Aufschlüsselung des Vokalismus: Steht im ,zweiten Zimmer" des Hauses nur ein Vokalbuchstabe $(\langle\mathrm{R} u\rangle$ in $\langle$ Ruder $\rangle)$, kann er sich ausbreiten - er wird lang und gespannt realisiert; steht dort ein Paar aus Vokal- und Konsonantbuchstabe ( $<H u n>$ in $<H u n d e>$ ), kann sich der Vokal nicht ausbreiten - er wird kurz und ungespannt realisiert. Der Reduktionssilbennukleus wird durch seine Position in der Garage als zu reduzierender Vokal sichtbar gemacht.

Nicht eigens modelliert wird im vorliegenden Modell die morphologische Struktur; die Ableitung morphologischer Schreibungen muss mit eigenen Mitteln bearbeitet werden (Röber 2009, S. 245 ff.).

Ansetzend am Vorschlag von Röber hat Bredel (2009) ein Modell zur Diskussion gestellt, in dem die Silben-/Fußstruktur und die morphologische Struktur simultan sichtbar gemacht werden. Silben- und Fußstrukturen sind analog zu Röber Modell und unter Beachtung des verzweigenden Nukleus (vgl. Abb. 3) strukturiert; der Morphemschnitt ist zusätzlich durch Einfärbung markiert (Abb. 7).

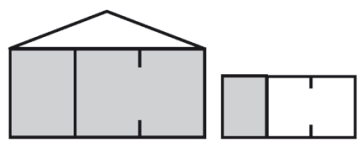

Abb. 7: Didaktisches Basismuster nach Bredel (2009)

In jüngerer Zeit hat Pracht (2012) einen Ansatz zur Diskussion gestellt, der sich auf neuere lerntheoretische Annahmen stützt. Hintergrund ist neben der orthographietheoretischen Orientierung am trochäischen Basismuster 
und basaler morphologischer Strukturen (Präfigierung, Flexion), die jedoch, soweit ich es erkennen kann, nicht konsequent visualisiert werden, eine schemabasierte Konzeptualisierung von Erwerbsprozessen: Prototypische Muster einer Sprache, so die Annahme, werden kognitiv schematisiert und dienen als Orientierungs- und Ausgangspunkt für die Entdeckung von Gesetzmäßigkeiten, die durch Übertragung weiterverarbeitet werden und so ganze Paradigmen konfigurieren. Pracht geht davon aus, dass „kompetente, gut funktionierende Schrift-/Sprachverarbeitung [...] in hohem Maße auf einem gut ausgebauten Netzwerk verlässlich verfügbarer und flexibel verwendbarer schrift-/sprachlicher Schemata [beruht]“ (ebd., S. 6). Auf dieser Grundlage arbeitet sie schemabildende Strukturen der Schriftsprache heraus: Am Anfang steht das trochäische Basismuster, das bei Pracht wie in Abbildung 8 visualisiert wird. Die Kreisgrößen symbolisieren die Betonungsverhältnisse; die Lerner/innen haben zuvor mit großen und kleinen Steinen die Betonung von Wörter gelegt und übertragen diese Visualisierung nun auf den Schreibprozess.

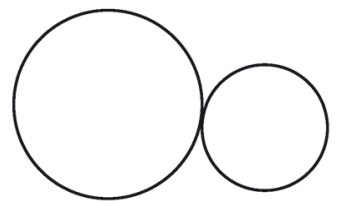

Abb. 8: Didaktisches Basismuster nach Pracht (2012)

Ausgehend davon, dass die Deutsche Orthographie ,gesprochensprachliche Muster gleichsam visualisiert und expliziert" (ebd., S. 9), argumentiert Pracht, dass dieses Lernangebot nicht nur den Schriftspracherwerb unterstützt, sondern dass gerade für Lerner/innen mit Deutsch als zweiter Sprache ,anhand der schriftsprachlichen Muster auch die Strukturmuster der gesprochenen Sprache zugänglich und transparent werden, so dass [...] die Lernenden sich ein zunehmend differenziertes Bild von der Sprache machen können“ (ebd.).

\section{Die Schrift als Modell für die Lautsprache - Fazit}

Den Ausgangspunkt des vorliegenden Beitrags bildete die Annahme, dass die Arbeit an rhythmisch-prosodischen Mustern für Lerner/innen des Deutschen nicht nur für den Laut-, sondern auch für den Schriftspracherwerb grundlegend ist.

Gezeigt wurde, dass diese Arbeit von herkömmlichen, auf einer zirkulären Tradition aufruhenden Lese-/Schreiberwerbsmodellen nicht nur nicht gefördert, sondern sogar doppelt erschwert wird: Die einseitige Fixierung auf die segmentale Laut-Buchstaben-Beziehungen und die damit erforder- 
lich werdende „Pilotsprache“ aktiviert statt der Normalaussprache, an der die Akzent- und Rhythmusstrukturen erprobt werden können, einen Arbeitsakzent, der für die Erschließung der segmentalen Struktur gebraucht wird. Darüber hinaus gestattet es die ausschließlich an der Schreibrichtung ausgerichtete Schriftdidaktik nicht, die in der Orthographie abgelegten Rhythmus- und Akzentstrukturen sehen zu lernen.

Dass Lerner/innen mit türkischer Ausgangssprache mit den herkömmlichen Methoden auf - meist unerklärt bleibende - Lernhemmnisse stoßen, wurde auf der Basis der Gegenüberstellung der türkischen und deutschen Rhythmus- und Akzentstrukturen und deren Konsequenzen für die Erfassung und Analyse von Wörtern und ihrer Strukturen zur Diskussion gestellt.

Neuere Modelle, die Akzent- und Rhythmussensitivität sowohl der gesprochenen als auch der geschriebenen Sprache aufgreifen und für den (Schrift-)Spracherwerb produktiv machen, wurden in Kapitel 6 vorgestellt.

Durch die Priorisierung der Lese- vor der Schreibrichtung und durch Orientierung am deutschen Kernwortschatz und hier am trochäischen Basismuster (als Grundlage für die Schemabildung) sowie durch die Visualisierung von Vokalqualitäten und -quantitäten ergeben sich daraus gerade für Lerner/innen mit Türkisch als Erstsprache folgende Lerngelegenheiten:

Zum einen wird der Wortbegriff systematisch erarbeitet: Ein an der Orthographie ausgerichteter Schriftspracherwerb führt zum Aufbau einer kognitiven Repräsentation der trochäischen „Gussform“, der Wortgrenzen und der internen Struktur von Wörtern (lexikalische/grammatische Morpheme). Zum anderen können die Lerner/innen Silben- und Akzentmuster des Deutschen, genauer: die Vokalquantität/-qualität in der Hauptsilbe, die Vokalreduktion in der Reduktionssilbe und die silbeninterne Phono-/Graphotaktik systematisch ableiten.

Zentral ist dabei, dass die Lerner/innen die Struktur der Schrift durch die Priorisierung der Leserichtung nicht nur für die Ermittlung der Wortschreibung, sondern zugleich für die Ermittlung der prosodischen Muster des Deutschen nutzen können: Sie können sehen lernen, was sie (ohne Unterstützung) nicht hören können.

Noch stehen profunde Analysen über die Wirksamkeit der hier vorgeschlagenen orthographiebasierten Lese-/Schreiblehrgänge aus. Einige qualitative Untersuchungen zeigen jedoch, dass nicht nur Kinder mit Türkisch, sondern auch Kinder mit Deutsch als Erstsprache von einem in dieser Weise strukturierten Zugriff auf das Schriftsystem profitieren (Röber 2006; Weinhold 2010; Krauß 2010).

Bei quantitativen Studien erhalten wir widersprüchliche Ergebnisse: Während Rautenberg (2012) bei Kindern, die silbenbasiert unterrichtet wurden, gegenüber solchen, die lautbasiert unterrichtet wurden, einen deutli- 
chen Vorsprung sieht, waren die Unterschiede bei Weinhold (2009) nur in Bezug auf die Lesefähigkeiten, nicht aber in Bezug auf die Rechtschreibleistungen signifikant.

Eine empirische Herausforderung für die Zukunft ist die Entwicklung von Testmaterialien, die auf einer sachgerechten Modellierung der Rechtschreibkompetenz beruhen (Blatt/Frahm 2011): Denn nicht nur die aktuell praktizierten Lese-Schreibmethoden, sondern auch die auf dem Markt befindlichen standardisierten Lese-/Rechtschreibtests basieren auf der Annahme, das Deutsche sei zugrundeliegend eine Lautschrift. So wird etwa die Richtigschreibung von „lautgetreuen Wörtern“ getestet - Wortmaterial, das auf eine bestimmte Schrifttheorie verweist bzw. diese zur Grundlage für die Modellierung der Rechtsschreibkompetenz macht, die orthographie- und lerntheoretisch in die falsche Richtung weisen.

\section{Literatur}

Abercrombie, David (1967): Elements of general phonetics. Edinburgh.

Ashby, Jane (2006): Prosody in skilled silent reading: evidence from eye movements. In: Journal of Research in Reading 29, S. 318-333.

Auer, Peter (2001): Silben- und akzentzählende Sprachen. In: Haspelmath, Martin et al. (Hg.): Sprachtypologie und sprachliche Universalien. Ein internationales Handbuch. 2. Halbbd. (= Handbücher zur Sprach- und Kommunikationswissenschaft 20). Berlin/New York, S. 1391-1399.

Auer, Peter/Uhmann, Susanne (1988): Silben- und akzentzählende Sprachen. Literaturüberblick und Diskussion. In: Zeitschrift für Sprachwissenschaft 7, S. 214-259.

Becker, Tabea (2008): Modelle des Schriftspracherwerbs: Eine kritische Bestandsaufnahme. In: Didaktik Deutsch 25, S. 78-95.

Becker, Tabea (2011): Schriftspracherwerb in der Zweitsprache: eine qualitative Längsschnittstudie. Baltmannsweiler.

Berg, Kristian/Fuhrhop, Nanna (2011): Komplexe Silbenkernschreibungen im Englischen im Vergleich mit dem Deutschen. In: Linguistische Berichte 228, S. 443-466.

Birk, Elisabeth/Häffner, Sonja (2005): Was ist phonologische Bewusstheit? Schrifttheoretische Analyse einer psychologischen Fragestellung. In: Huneke, Hans-Werner (Hg.): Geschriebene Sprache. Strukturen, Erwerb, didaktische Modellbildungen. (= Schriftenreihe der Pädagogischen Hochschule Heidelberg 45). Heidelberg, S. 53-72.

Blatt, Inge/Frahm, Sarah (2011): Rechtschreibtests. In: Bredel, Ursula/Reißig, Tilo (Hg.): Weiterführender Orthographieunterricht. (= Handbuch Deutschunterricht in Theorie und Praxis 5). Baltmannsweiler, S. 546-570.

Blumenstock, Leonhard (1993): Schriftspracherwerb: mit oder ohne Fibel? In: Haarmann, Dieter (Hg.): Handbuch Grundschule. Bd. 2: Fachdidaktik: Inhalte und Bereiche grundlegender Bildung. Weinheim/Basel, S. 81-99. 
Bredel, Ursula (2009): Orthographie als System - Orthographieerwerb als Systemerwerb. In: Zeitschrift für Literaturwissenschaft und Linguistik 39, 153, S. 135-154.

Bredel, Ursula/Müller, Astrid/Hinney, Gabriele (Hg.) (2011): Schriftsystem und Schrifterwerb: Linguistisch, didaktisch, empirisch. (= Reihe Germanistische Linguistik 289). Berlin/New York.

Bredel, Ursula/Siebert-Ott, Gesa/Thelen, Tobias (Hg.) (2004): Schriftspracherwerb und Orthographie. (= Diskussionsforum Deutsch 16). Baltmannsweiler.

Dauer, Rebecca M. (1983): Stress-timing and syllable-timing reanalyzed. In: Journal of Phonetics 11, S. 51-62.

Domahs, Ulrike et al. (2012): Processing (un-)predictable word stress: ERP evidence from Turkish. In: Language and Cognitive Processes, S. 1-20. Internet: www.tandfonline. com/doi/abs/10.1080/01690965.2011.634590 (Stand 13.06.2012).

Dupoux, Emmanuel et al. (1997): A destressing 'deafness' in French? In: Journal of Memory and Language 36, S. 406-421.

Eichler, Wolfgang (1976): Zur linguistischen Fehleranalyse von Spontanschreibungen bei Vor- und Grundschulkindern. In: Hofer, Adolf (Hg.): Lesenlernen: Theorie und Unterricht. (= Sprache und Lernen 44). Düsseldorf, S. 246-264.

Eisenberg, Peter (2006): Grundriss der deutschen Grammatik. Bd. 1: Das Wort. 3., durchges. Aufl. Stuttgart u.a.

Frith, Uta (1985): Beneath the surface of developmental dyslexia. In: Patterson, Karalyn E./Marshall, John C./Coltheart, Max (Hg.): Surface dyslexia: neuropsychological and cognitive studies of phonological reading. London, S. 301-330.

Glück, Christian/Obergföll, Katharina (2009): „ein-dei-dei (eins-zwei-drei)“ - Diagnostik des phonologischen Arbeitsgedächtnisses bei aussprachegestörten Kindern. In: Die Sprachheilarbeit 54, 4, S. 138-145.

Grimm, Hannelore (2003): Störungen der Sprachentwicklung: Grundlagen - Ursachen - Diagnose - Intervention - Prävention. 2., überarb. Aufl. Göttingen u.a.

Gut, Ulrike/Trouvain, Jürgen/Barry, William (2007): Bridging research on phonetic descriptions with knowledge from teaching practice - The case of prosody in non-native speech. In: Gut, Ulrike/Trouvain, Jürgen (Hg.): Non-native prosody. Phonetic description and teaching practice. (= Trends in Linguistics: Studies and Monographs 186). Berlin/New York, S. 5-21.

Hinney, Gabriele (1997): Neubestimmung von Lerninhalten für den Rechtschreibunterricht. Ein fachdidaktischer Beitrag zur Schriftaneignung als Problemlöseprozess. Frankfurt a.M. u.a.

Hinney, Gabriele (2010): Wortschreibungskompetenz und sprachbewusster Unterricht. Eine Alternativkonzeption zur herkömmlichen Sicht auf den Schriftspracherwerb. In: Bredel/Müller/Hinney (Hg.), S. 47-100.

Huneke, Hans-Werner (2008): Was Kinder vor der Schule können sollen. Schriftvorbereitende Kompetenzen in Bildungsplänen der Bundesländer für Kindertageseinrichtungen - ein Vergleich. Internet: www.symposion-deutschdidaktik.de/fileadmin/template/ download/beitraege/praesentationen_vortraege/08Huneke.pdf (Stand 13.06.2012). 
Jeuk, Stefan (2007): Schriftaneignung und Grammatisierung bei mehrsprachigen Kindern im Anfangsunterricht. In: Haueis, Eduard/Schallenberger, Stefan (Hg.): (Schrift-)Spracherwerb und Grammati(kali)sierung. (= Osnabrücker Beiträge zur Sprachtheorie 73). Duisburg u.a., S. 34-55.

Kaltenbacher, Erika (1998): Zum Sprachrhythmus des Deutschen und seinem Erwerb. In: Wegener, Heide (Hg.): Eine zweite Sprache lernen. Empirische Untersuchungen zum Zweitsprachenerwerb. (= Tübinger Beiträge zur Linguistik, Serie A, 24). Tübingen, S. 21-38.

Krauß, Andrea (2010). Orthographieerwerb von Beginn an. Ein silbenorientiertes Konzept für den Anfangsunterricht. In: Bredel/Müller/Hinney (Hg.), S. 133-150.

Maas, Utz (1992): Grundzüge der deutschen Orthographie. (= Reihe Germanistische Linguistik 120). Tübingen.

Marx, Harald (1992): Methodische und inhaltliche Argumente für und wider eine frühe Identifikation und Prädiktion von Lese-Rechtschreibschwierigkeiten. In: Diagnostica 38, S. 249-268.

Metze, Wilfried (2008): Lernwegsorientierter Schriftspracherwerb im Spiegel der Empirie und des Schulalltags. Internet: www.wilfriedmetze.de/Vortrag_Zurich_31.5.08.pdf (Stand 13.06.2012).

Meyer-Ingwersen, Johannes/Neumann, Rosemarie/Kummer, Matthias (1977): Zur Sprachentwicklung türkischer Schüler in der Bundesrepublik. Band 1. (= ScriptorTaschenbücher, Literatur + Sprache + Didaktik 105). Kronberg i.Ts.

Noack, Christina (2004): Lesekompetenzen von badischen und norddeutschen Hauptschülern. Ein phonologisches Analysekonzept zur Dekodierfähigkeit. In: Bredel/ Siebert-Ott/Thelen (Hg.), S. 104-122.

Penner, Zvi (2007): Vom Sprachrhythmus zur Rechtschreibung. Die sprachrhythmischen Grundlagen der deutschen Orthographie. (= Sprachkompetent für die Schule). Bern.

Pracht, Henrike (2012): Schemabasierte Basisalphabetisierung im Deutschen. Ein Praxisbuch für Lehrkräfte. Münster.

Primus, Beatrice/Evertz, Martin (2011): The graphematic foot in English and German. Manuskript. Köln

Rautenberg, Iris (2012): Musik und Sprache. Eine Längsschnittstudie zu Effekten musikalischer Förderung auf die schriftsprachlichen Leistungen von GrundschülerInnen. Baltmannsweiler.

Reichen, Jürgen (1988): Lesen durch Schreiben. Heft 1: Wie Kinder selbstgesteuert Lesen lernen: lesedidaktische, lernpsychologische und schulpädagogische Grundlagen eines vom Schüler selbstgesteuerten Schriftspracherwerbs. 3. Aufl. Hamburg.

Reichen, Jürgen (2001): „Hannah hat Kino im Kopf“: Die Reichen-Methode „Lesen durch Schreiben“ und ihre Hintergründe für LehrerInnen, Studierende und Eltern. Hamburg.

Richter, Julia (2008): Phonetische Reduktion im Deutschen als L2. Eine empirische Querschnittsstudie. (= Perspektiven Deutsch als Fremdsprache 22). Baltmannsweiler. 
Röber, Christa (2006): Die Systematik der Orthographie als Basis von Analysen von Kinderschreibungen. Eine empirische Untersuchung zur Schreibung der i-Laute. In: Bredel, Ursula/Günther, Hartmut (Hg.): Orthographietheorie und Rechtschreibunterricht. (= Linguistische Arbeiten 509). Tübingen, S. 71-102.

Röber, Christa (2009): Die Leistungen der Kinder beim Lesen- und Schreibenlernen. Grundlagen der Silbenanalytischen Methode. Ein Arbeitsbuch mit Übungsaufgaben. Baltmannsweiler.

Röber-Siekmeyer, Christa (1997): Die Schriftsprache entdecken. Rechtschreiben im Offenen Unterricht. 3., erg. u. neu ausgest. Aufl. (= Beltz Praxis; Reihe Werkstattbuch Grundschule). Weinheim.

Scheerer-Neumann, Gerheid (1998). Schriftspracherwerb: „The State of the Art“ aus psychologischer Sicht. In: Huber, Ludowika/Kegel, Gerd/Speck-Hamdan, Angelika (Hg.): Einblicke in den Schriftspracherwerb. Braunschweig, S. 31-46.

Schneider, Wolfgang/Roth, Ellen/Küspert, Petra (1999). Möglichkeiten der frühen Prävention von Lese-Rechtschreibproblemen: Das Würzburger Trainingsprogramm zur Förderung sprachlicher Bewußtheit bei Kindergartenkindern. In: Kindheit und Entwicklung 8, S. 147-152.

Steinig, Wolfgang et al. (2009): Schreiben von Kindern im diachronen Vergleich. Texte von Viertklässern aus den Jahren 1972 und 2002. Münster u.a.

Störmer, Maike (2008): Fibel und/oder Anlauttabelle. Gibt es noch einen Methodenstreit in der Grundschule? Internet: www.uni-koeln.de/phil-fak/deutsch/lehrende/schindler/ downloads/files/Examensarbeit.Methodenstreit-Fibel-Anlauttabelle.pdf (Stand 20.5.2012).

Thomé, Günther (1987): Rechtschreibfehler türkischer und deutscher Schüler. (= Sammlung Groos 33). Heidelberg.

Thomé, Günther (1999): Orthographieerwerb: Qualitative Fehleranalysen zum Aufbau der orthographischen Kompetenz. (= Theorie und Vermittlung der Sprache 29). Frankfurt a.M. u.a.

Thomé, Günther (2003): Entwicklung der basalen Rechtschreibkenntnisse. In: Bredel, Ursula et al. (Hg.): Didaktik der deutschen Sprache. Ein Handbuch. Bd. 1. (= UniTaschenbücher 8235; Pädagogik, Sprachwissenschaft). Paderborn, S. 369-379.

Valtin, Renate (1997): Stufen des Lesen- und Schreibenlernens. Schriftspracherwerb als Entwicklungsprozeß. In: Haarmann, Dieter (Hg.): Handbuch Grundschule. Bd. 2: Fachdidaktik: Inhalte und Bereiche grundlegender Bildung. 3., aktual. u. neu ausgest. Aufl. Weinheim/Basel, S. 76-88.

Vogt, Miriam (2004): Gurke gesehen, /g...goA:::k.k.kE::/ gesprochen und <Gake> geschrieben: Dokumentation und Analyse von Schreibprozessen mit Hilfe von Transkripten. In: Bremerich-Vos, Albert/Löffler, Cordula/Herné, Karl-Ludwig (Hg.): Neue Beiträge zur Rechtschreibtheorie und -didaktik. Festschrift für Carl Ludwig Naumann zum 60. Geburtstag. Freiburg, S. 125-144.

Wängler, Hans-Heinrich (1974): Grundriss einer Phonetik des Deutschen: mit einer allgemeinen Einführung in die Phonetik. 3., durchges. Aufl. Marburg.

Weber, Christiane et al. (2004): Discrimination of word stress in early infant perception: electrophysiological evidence. In: Cognitive Brain Research 18, S. 149-161. 
Weinert, Franz E. (1996): Lerntheorien und Instruktionsmodelle. In: Weinert, Franz E. (Hg.): Psychologie des Lernens und der Instruktion. (= Enzyklopädie der Psychologie: Themenbereich D; Praxisgebiete; Serie 1; Pädagogische Psychologie 2). Göttingen u.a., S. 1-118.

Weingarten, Rüdiger (2004): Die Silbe im Schreibprozess und im Schriftspracherwerb. In: Bredel/Siebert-Ott/Thelen (Hg.), S. 6-21.

Weinhold, Swantje (2009): Effekte fachdidaktischer Ansätze auf den Schriftspracherwerb in der Grundschule. Lese- und Rechtschreibleistungen in den Jahrgangsstufen 1-4. In: Didaktik Deutsch 27, S. 53-73.

Weinhold, Swantje (2010): Silben sind besser als Laute und Buchstaben. Ergebnisse einer Untersuchung kontroverser Methoden für den Schriftspracherwerb. In: Grundschulmagazin 4, S. 11-15. 